\title{
Capillary breakup extensional rheometry (CaBER) of sodium carboxymethylcellulose solutions in water and propylene glycol/water mixtures
}

\author{
Sylwia Różańska, ${ }^{1}$ Karel Verbeke, ${ }^{2}$ Jacek Różański, ${ }^{1}$ Christian Clasen, ${ }^{2}$ Patrycja Wagner ${ }^{1}$ \\ ${ }^{1}$ Department of Chemical Engineering and Equipment, Faculty of Chemical Technology, Poznan \\ University of Technology, Berdychowo 4, PL 61-131 Poznan, Poland \\ ${ }^{2}$ Department of Chemical Engineering, KU Leuven, Celestijnenlaan 200 F, 3001 Leuven, Belgium \\ Correspondence to: S. Różańska (E-mail: sylwia.rozanska@put.poznan.pl)
}

((Additional Supporting Information may be found in the online version of this article.))

\begin{abstract}
This paper presents the results of CaBER experiments conducted for semidilute solutions of carboxymethylcellulose sodium salt (Na-CMC) with degrees of substitution (DS) ranging from 0.62 to 1.04 in distilled water and propylene glycol (PG)/water mixtures. The partial aggregation of Na-CMC chains with DS $<1$ observed in aqueous solutions triggers an increase in apparent extensional viscosity and extension of break-up time. The rheological properties of $\mathrm{Na}-\mathrm{CMC}$ solutions in propylene glycol/water mixture are determined by the solubility of the polymer and the physical crosslinking of chains. The disappearance of the elasto-capillary regime during the filament thinning of $\mathrm{Na}-\mathrm{CMC}$ solutions with DS $<1$ in propylene glycol/water mixture was linked to the physical crosslinking of polymer chains. The shape of the extensional viscosity curve for $\mathrm{Na}-\mathrm{CMC}$ solutions with DS $=1.04$ in PG/water mixture was characteristic for semidilute polymer solutions with a low number of entanglements.
\end{abstract}

KEYWORDS: extensional viscosity, capillary thinning, extensional relaxation time, CaBER

\section{INTRODUCTION}

Sodium carboxymethylcellulose (Na-CMC) is the most important water-soluble anionic cellulose derivative, as it is widely used as a viscosity modifier, emulsion stabilizer, drug reducing agent, and water-retention agent in pharmaceutical, food, paper, paint and cosmetics industries. ${ }^{1-4}$ In a number of technical processes polymer solutions are subjected not only to shearing but also to extensional deformations, as for example in batch filling operations, flow through porous media, flow through converging channels, spray and atomization processes, mixing or drag reduction. ${ }^{5-15}$ Consequently, the results of rheological tests in the extensional flow can be helpful for designing industrial systems.
The rheological properties of aqueous $\mathrm{Na}-\mathrm{CMC}$ solutions under shear flow are well understood, however, relatively few data have been published to date on the properties of solutions of this polyelectrolyte in an extensional flow. ${ }^{7,16-}$ 20 In addition, most of the study findings published so far have been obtained using methods without pure extension (opposed jet and entrance flow devices)..$^{7,16,17,19}$ Based on these experiments it was found that the extensional viscosity, similarly to the shear viscosity of aqueous $\mathrm{Na}-\mathrm{CMC}$ solutions, depends not only on the average molecular weight, but also on the degree of substitution DS (average number of carboxymethyl groups substituted per anhydroglucose at the 2-, 3-, and 6-positions) and salt concentration. ${ }^{21}$ 
A purely extensional flow of a low viscosity liquid can be achieved with the capillary thinning of a liquid filament (within a so-called capillary break-up extensional rheometer (CaBER)). The experiment involves rapid separation of two plates between which a fluid sample is placed. After that, the plates are kept at a constant distance, while monitoring the evolution of the mid-filament diameter $\left(D_{\text {mid }}\right)$ of the cylindrical liquid bridge during the process of its necking and breakup.

The CaBER method to measure the extensional behavior of sodium carboxymethylcellulose aqueous solutions was used by Zhao et al. ${ }^{19}$ and Choi et al. ${ }^{18}$ Zhao et al. ${ }^{19}$ determined that the values of the filament break-up time increase with increasing of weight average molecular weight $\left(M_{w}\right)$, which has been attributed to an increase of the extensional viscosity of the $\mathrm{Na}$ CMC solutions. Choi et al. ${ }^{18}$ analyzed the effect of saliva on the extensional rheological properties of aqueous solutions of xanthan gum and $\mathrm{Na}-\mathrm{CMC}$. The addition of saliva increased the filament break-up time for xanthan gum, while it had little effect on the break-up time of the NaCMC filament.

The measurement results obtained using extensional rheometry based on capillary breakup dynamics are presented in the literature for several aqueous solutions of polysaccharides ${ }^{18,22-29}$, however there are only four studies analyzing the effect of solvent quality on the process of filament thinning of semidilute polysaccharide solutions as well as synthetic polymer solutions. ${ }^{28-31}$

De Dier et $a .^{28}$ studied schizophyllan, which takes on two different configurations in solution: a random coil state in dimethyl sulfoxide (DMSO) and a more rigid triple-helical structure in aqueous solution. The authors demonstrated that the extensional relaxation time increased with increasing chain rigidity, and this relative increase became stronger with increasing polymer concentration. Sharma et al. $^{29}$ presented the shear and extensional rheology of aqueous solutions of ethyl hydroxyethyl cellulose (EHEC), and its hydrophobically modified analogue (hmEHEC). The semidilute solutions of hmEHEC exhibit extensional thinning, while the unmodified bare chains of EHEC display an increase in extensional viscosity, up to a plateau value. The reduction in extensional viscosity with increasing extension rate for the hydrophobically modified cellulose ether has been explained by the disruption of a transient elastic network that is initially formed by the intermolecular association of hydrophobic stickers. Jimenez et al. $^{30}$ characterized the extensional rheology of two model polyelectrolytes (poly(sodium 4styrenesulfonate) (NaPSS) and poly(acrylic acid) (PAA)) in two different solvents (deionized water and glycerol/water mixtures) by using drippingonto-substrate (DoS) rheometry. Unlike the shear relaxation time, which decreases with increases in concentration in unentangled, semidilute solutions, the extensional relaxation time of PAA solutions increases with an exponent of $1 / 2$, and the entangled semidilute solutions also exhibit a stronger concentration dependence of $3 / 2$. Walter et al. ${ }^{31}$ analyzed the effect of salt on the extensional rheological response of aqueous solutions of partially hydrolyzed polyacrylamide (HPAM). An increase in monovalent salt concentration led to a decrease in extensional relaxation times, while an increase in divalent salt concentration caused an increase in extensional relaxation time. Divalent cations may form transient complexes with the polyelectrolyte chain, causing higher extensional relaxation times at high divalent salt concentrations.

This paper presents the results of CaBER experiments for aqueous solutions of $\mathrm{Na}-\mathrm{CMC}$ and $\mathrm{Na}-\mathrm{CMC}$ solutions in propylene glycol $(\mathrm{PG}) /$ water mixtures. Komorowska et al. ${ }^{32}$ observed a strong synergism between molecules of Na-CMC with a DS of 0.62 and 0.79 , and propylene glycol. At the same time, Na-CMC solutions with a DS $=1.04$ demonstrated no intermolecular crosslinking of polymer chains in PG/water mixture. The main aim of the study was therefore to determine the actual effect of 
the degree of substitution on the extensional rheological properties of $\mathrm{Na}-\mathrm{CMC} / \mathrm{PG} /$ water mixtures during the capillary thinning of filaments.

\section{THEORY OF CAPILLARY THINNING EXTENSIONAL RHEOMETRY}

During a capillary thinning experiment, a liquid bridge configuration is generated by rapidly separating two cylindrical plates, between which the fluid is held, over a small distance, and the subsequent evolution of the mid-filament diameter $D_{\text {mid }}$ of the created liquid filament with time $t$ is recorded. Based on the dependency $D_{\text {mid }}$ $=f(t)$, it is possible to calculate the extension rate within the filament:

$$
\dot{\varepsilon}=-\frac{2}{D_{\text {mid }}} \frac{d D_{\text {mid }}}{d t}
$$

and an apparent extensional viscosity. ${ }^{33}$

$$
\eta_{E, a p p}=-\frac{\Gamma}{d D_{\text {mid }} / d t}
$$

To calculate $\eta_{\mathrm{E} \text {,app, }}$ it is necessary to make an independent measurement of the surface tension $\Gamma$.

The thinning of the filament is controlled by a balance between inertial, viscous, elastic, gravitational and capillary forces. ${ }^{34}$ The trade-off between the effect of inertia with respect to viscous and elastic forces during capillary thinning can be determined based on the Ohnesorge number:

$$
O h=\frac{\eta}{\sqrt{\rho \Gamma\left(D_{\text {mid }} / 2\right)}}
$$

and the Deborah number respectively:

$$
D e=\frac{\lambda}{\sqrt{\frac{\rho\left(D_{\text {mid }} / 2\right)^{3}}{\Gamma}}}
$$

where $\rho$ is the density, $\eta$ is the viscosity and $\lambda$ is the fluid's longest relaxation time. Inertia can be neglected for values of the Ohnesorge number Oh $>1$ and Deborah number De $>1 .^{6,34}$ In case of entangled polymer solutions, inertia usually has little effect on the evolution of the filament diameter due to the relatively large viscosity and thus large Ohnesorge number. Gravitational effects on the fluid bridge/liquid filament might manifest in an asymmetry of the liquid filament with respect to the middle plane parallel to the disks. Such gravitational sagging can be observed in the initial period of capillary thinning (regime I), but becomes negligible when values of the local Bond number

$$
B o=\frac{g \rho D_{m i d}^{2}}{4 \Gamma}
$$

are lower than $0.2^{35}$, where $g$ is the gravitational constant. During the further evolution of the mid-filament diameter (for Bo >0.2), three more regimes, characteristic for entangled polymer solutions, can be distinguished. ${ }^{34,35}$

Regimes II and III of the filament thinning are controlled by a visco-capillary balance. Initially, at large diameters $D_{\min }$, the capillary pressure balancing the viscous stresses is low, thus resulting in a low extension rate $\dot{\varepsilon}$ and a Newtonian thinning response for which the midfilament diameter decreases linearly in time following. ${ }^{36,37}$

$$
D_{\text {mid }}(t)=0.1418 \cdot \frac{\Gamma}{\eta_{0}}\left(t_{c}-t\right)
$$

Here $\eta_{0}$ is the zero shear viscosity and $t_{c}$ is the time to breakup. As thinning proceeds, the capillary pressure and thus the extension rate $\dot{\varepsilon}$ increases and eventually disentanglement and orientation of the polymer chains occurs (regime III). Filament thinning is still controlled by a viscocapillary balance, but the viscosity exhibits a power law dependency on the extension rate. ${ }^{38}$

$$
D_{\text {mid }}(t)=2 \Phi \cdot \frac{\Gamma}{k}\left(t_{c}-t\right)^{n}
$$


where $k$ and $n$ are the parameters of a powerlaw model and $\Phi$ is numerical front factor which is a function of $n$.

$\eta_{E}=k \cdot \dot{\varepsilon}^{n-1}$

Between the regimes II and III described by equations (6) and (7) there is a transitional range in which the extensional viscosity decreases, but that is usually not captured by a power law.

At high extension rates viscous stresses become negligible compared to the increasing contribution of elastic (polymeric) stresses, and eventually elastic and capillary contributions to the total force balance each other (regime IV).

Clasen $^{35}$ and Anna and McKinley ${ }^{33}$ used a local elasto-capillary number to determine the boundary between regimes III and IV.

$$
E c=\frac{2 \cdot \lambda \Gamma}{\eta D_{\text {mid }}(t)}
$$

Generally, thinning is controlled by the overall elasticity for $E c>>1$, and by viscosity for $E c<<1$, with critical transition value of $\mathrm{Ec}=4.7$.

For the elasto-capillary regime Entov and $\mathrm{Hinch}^{39}$ showed that for a multi-mode FENE fluid the diameter will decrease exponentially with time:

$$
D_{\text {mid }}(t)=D_{0}\left(\frac{G D_{0}}{4 \Gamma}\right)^{1 / 3} e^{-\frac{t}{3 \lambda_{E}}}
$$

where $G$ is the elastic modulus of the fluid and $\lambda_{E}$ is the longest extensional relaxation time.

As presented by Entov and Hinch ${ }^{39}$ as well as Anna and McKinley ${ }^{33}$, the diameter will approach a linear decay at late times. This is caused by the polymer chains approaching their fully stretched state, and the elastic stresses can no longer grow to resist the increasing capillary pressure. Anna and McKinley $^{33}$ suggested to describe the transition between exponential and linear decay of the midfilament diameter with the following equation:
$D_{\text {mid }}(t)=A e^{-B t}-C t+D$

where $A, B, C$, and $D$ are fitting parameters. The fitting parameters have the physical relevance. ${ }^{40,41}$ Parameter $B$ is directly related to the extensional relaxation time $\left(B=1 /\left(3 \lambda_{\mathrm{E}}\right)\right)$, while parameter $C$ is used in the current paper to describe the extensional viscosity at late times, $\eta_{\mathrm{E}, \infty}\left(C=\Gamma / \eta_{\mathrm{E}, \infty}\right)$.

\section{EXPERIMENTAL}

\section{Materials}

Three sodium carboxymethylcellulose (Na-CMC) with different degrees of substitution ( $D S=0.7$, 0.9 and 1.2 - based on manufacturer's data) and with a similar weight average molecular weight $\left(M_{w}=250,000 \mathrm{~g} / \mathrm{mol}\right.$ - based on manufacturer's data) were obtained from Sigma-Aldrich (Poland). The same Na-CMC samples were investigated in the works Komorowska et al. ${ }^{32}$ and Różańska and Różański ${ }^{17}$ (taken from the same bottle). The DS values specified by the manufacturer were verified using a standard method. ${ }^{41,42}$ The obtained DS values were lower than those specified by the manufacturer and determined as $0.62,0.79$ and 1.04 , respectively. To distinguish between polymers with different degrees of substitution the following notations are used: $\mathrm{Na}-\mathrm{CMC}_{0.62}$ for $\mathrm{DS}=0.62 ; \mathrm{Na}-\mathrm{CMC}_{0.79}$ for $\mathrm{DS}=0.79$; and $\mathrm{Na}-\mathrm{CMC}_{1.04}$ for $\mathrm{DS}=1.04$. Measurements of the molecular weight distributions performed by size-exclusion chromatography show that the polydispersity indexes $\left(\mathrm{PDI}=M_{w} / M_{n}\right)$ are similar in value as for the sodium carboxymethylcellulose used in the earlier studies (for DS 0.62; 0.79 and 1.04 they are equal to $2.09\left(M_{w}=264,400 \mathrm{~g} / \mathrm{mol}\right) ; 1.95$ $\left(M_{w}=242,200 \mathrm{~g} / \mathrm{mol}\right)$ and $1.83\left(M_{w}=262,400\right.$ $\mathrm{g} / \mathrm{mol}$ ), respectively). Propylene glycol (PG) used in the current study was supplied by Donauchem (Poland, Rokietnica/Poznań) with a purity of 99.94 wt\% (PG for pharmaceutical use).

\section{Preparation of the solutions}

The filament thinning experiments were carried out for solutions with concentrations of PG 
ranging from $40 \mathrm{wt} \%$ to $90 \mathrm{wt} \%$ and $\mathrm{Na}-\mathrm{CMC}$ from $1.4 \mathrm{wt} \%$ to $2.2 \mathrm{wt} \%$. The procedure for the preparation of the solutions is described in the work of Komorowska et $a I^{32}$. Depending on the concentration of $\mathrm{Na}-\mathrm{CMC}$ and propylene glycol, the solution preparation was done in two different ways. All solutions with concentrations below 1.6 wt\% of $\mathrm{Na}-\mathrm{CMC}$ and 80 wt\% of PG were prepared at room temperature. The required amount of $\mathrm{Na}-\mathrm{CMC}$ was added gradually to a beaker with an exactly measured amount of distilled water. The solutions were mixed using a magnetic stirrer for 24 hours to ensure complete hydration of the Na-CMC. Then the PG was gradually added and the obtained mixture was stirred for $24 \mathrm{~h}$.

The solutions with the $\mathrm{Na}-\mathrm{CMC}$ concentration of greater than or equal to $1.6 \mathrm{wt} \%$ and the PG concentrations greater than or equal to $80 \mathrm{wt} \%$ obtained through the above procedure were heterogeneous, and were therefore prepared according to the method detailed in the following. As the initial step, solutions containing lower $\mathrm{Na}-\mathrm{CMC}$ and PG concentrations were prepared identically to the first procedure. In a next step, the obtained $\mathrm{Na}-\mathrm{CMC} / \mathrm{PG} /$ water mixture was heated to a temperature of $40^{\circ} \mathrm{C}$ and the excess of water was slowly evaporated. During evaporation, the sample was constantly stirred and weighed in order to achieve the specific Na-CMC and PG concentrations. The time needed to evaporate the water depends on the concentrations of $\mathrm{Na}-\mathrm{CMC}$ and PG (from 8 to 14 hours).

Preliminary rheological measurements showed the $\mathrm{Na}-\mathrm{CMC} / \mathrm{PG} /$ water solutions to be timedependent fluids. During the preparation samples were subjected to shear. As shown in Figure S1 (appendix), the reconstruction of their microstructure is very slow. Repeating measurements in approximately 24 hours have shown that after 9 days the results of the sweep strain experiment were fully repeated. It should be noted that the results were repeatable even after 30 days from the preparation of the solutions. On that basis, the period between sample preparation and measurements was set at 12 days (in practice, 12 to 17 days after preparation).

The stability of the rheological properties of $\mathrm{Na}$ $\mathrm{CMC}$ solutions was also controlled using an opposed nozzle device (described in detail in Różańska et $a l .{ }^{4}$ ). The results obtained in shear viscosity and apparent extensional viscosity measurements for aqueous solutions of $\mathrm{Na}-\mathrm{CMC}$ stirred for $24 \mathrm{~h}$ and $48 \mathrm{~h}$ were convergent, which confirms the achievement of the maximum polysaccharide hydration (Figure S2, appendix). Studies on aqueous solutions were carried out 48 hours after the preparation.

\section{Rheological measurements}

All rheological experiments were performed with a stress controlled rheometer (Physica MCR 501, Anton-Paar) at $20{ }^{\circ} \mathrm{C}$. Small amplitude oscillatory shear measurements were performed using $60 \mathrm{~mm}$ diameter parallel plates (gap 1 $\mathrm{mm}$ ). After loading, the sample was kept at rest for 4 minutes in order to eliminate residual stress histories. The linear viscoelastic range of each solution was identified by on oscillatory strain sweep, which was conducted by increasing the strain amplitude $\gamma_{0}$ from $0.01 \%$ to $1000 \%$ at a constant frequency of $1 \mathrm{~Hz}$. The range of linear viscoelasticity for all the solutions studied ended at a strain amplitude value $\gamma_{0} \geq 3 \%$. The frequency sweep experiments were conducted at $\gamma_{0}=1 \%$. The parameters obtained from the oscillatory test data were the storage modulus $G^{\prime}$ and the loss modulus $G^{\prime \prime}$. All rheological tests were performed at a stabilized temperature of $20 \pm 0.1^{\circ} \mathrm{C}$.

For the continuous shear flow rheological measurements, a cone-and-plate geometry (diameter - $59.974 \mathrm{~mm}$, angle - 2.014 , truncation - $254 \mu \mathrm{m}$ ) was used. The sample loading procedures were the same as those used in the SAOS tests described above.

Extensional rheological measurements were performed using a Capillary Breakup Extensional Rheometer (CaBER-1, Thermo Haake, Germany). 
The CaBER-1 was equipped with plates with a diameter $d=4 \mathrm{~mm}$ and the sample was loaded at an initial gap of $2 \mathrm{~mm}$. With a step stretch experiment to a final gap of $6 \mathrm{~mm}$, an unstable liquid bridge was created that was subsequently thinning under capillary action. A high-speed camera was used to record the evolution of capillary thinning bridge, with a temporal resolution down to $0.33 \mathrm{~ms} /$ frame and a spatial resolution of $1.9 \mu \mathrm{m} /$ pixel. Images taken this way were processed with an algorithm developed at $\mathrm{KU}$ Leuven in Matlab, to determine the edge of the filament and minimum the diameter $D_{\text {mid }}$ (filament diameter in its middle part) with subpixel accuracy. ${ }^{33}$

\section{Surface tension}

Equilibrium surface tension of the solutions was measured at $20 \pm 0.5{ }^{\circ} \mathrm{C}$ by using a Wilhelmy plate tensiometer (Krüss K9, Germany). The plate was cleaned with distilled water and flamed before each measurement. Three measurements were made for each solution.

\section{RESULTS AND DISCUSSION}

\section{Oscillatory shear flow}

Small amplitude oscillatory shear (SAOS) measurement results for sodium carboxymethylcellulose solutions in PG/water mixture with a PG concentration of $C_{P G} \geq 70 \%$ were discussed by Komorowska et al. ${ }^{32}$ The authors showed that the $\mathrm{Na}-\mathrm{CMC}_{0.62}$ and $\mathrm{Na}$ $\mathrm{CMC}_{0.79}$ chains undergo physical crosslinking, probably as a result of the formation of hydrogen bonds between $\mathrm{OH}$ groups of propylene glycol and $\mathrm{Na}-\mathrm{CMC}$. It was found that this crosslinked network was formed at a concentration of $c_{\mathrm{PG}} \geq 80 \%$. On the other hand, there is no crosslinking of the higher DS Na-CMC $\mathrm{CM}_{1.04}$ chains in PG/water mixtures. Figure 1 presents SAOS experiment results for $\mathrm{Na}-\mathrm{CMC}$ solutions with different degrees of substitution in a $50 \%$ PG solution. For the $\mathrm{Na}-\mathrm{CMC}_{1.04}$ solution the mechanical spectrum at low oscillation frequencies is characteristic for a terminal flow regime, whereas for $\mathrm{Na}-\mathrm{CMC}_{0.62}$ and $\mathrm{Na}-\mathrm{CMC}_{0.9}$ there are significant deviations from the indicated scaling exponents of 1 and 2 for $G$ " and $\mathrm{G}^{\prime}$ respectively, characteristic for monodisperse polymer solutions.

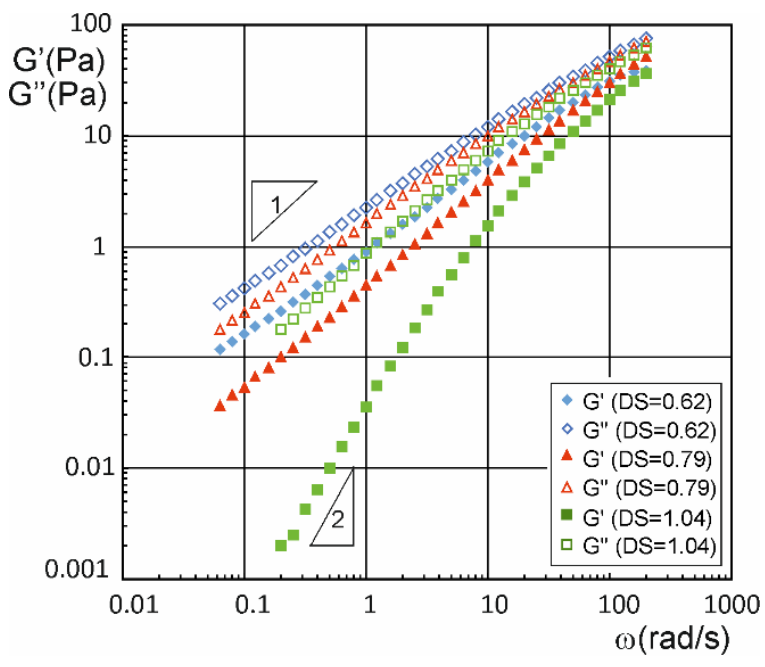

FIGURE 1 Mechanical spectra for solutions of $\mathrm{Na}$ CMC (1.6 wt\%) with different degrees of substitution (DS) in PG/water mixture (50 wt\% of PG)

In the case of sodium carboxymethylcellulose solutions with lower DS, scaling exponents have similar values and amount to $G^{\prime} \propto \omega^{0.73}$ and $G^{\prime \prime} \propto \omega^{0.75}$ for $\mathrm{Na}-\mathrm{CMC}_{0.62}$ and $G^{\prime} \propto \omega^{0.81}$ and $G^{\prime \prime} \propto \omega^{0.91}$ for $\mathrm{Na}-\mathrm{CMC}_{0.79}$, and over the entire range of oscillation frequencies $G^{\prime \prime}$ is larger than $G^{\prime}$. The mechanical spectra for $\mathrm{Na}-\mathrm{CMC}_{0.62}$ and $\mathrm{Na}-\mathrm{CMC}_{0.79}$ solutions in the PG/water mixture indicate a partial crosslinking of polyions close to a percolated structure, although the viscous contribution remains dominant over the entire frequency range.

Figure S7 (appendix) shows results of SAOS measurements for $\mathrm{Na}-\mathrm{CMC}_{1.04}$ solutions also in higher concentrated PG/water mixture $\left(c_{P G}=80 \%\right)$ with polymer concentrations of $1.4 \%$ up to $2.2 \%$. While the moduli curves exhibit a general increase with both polymer and PG concentration, they all exhibit a clear terminal flow regime with no indication of crosslinking.

\section{Concentration dependence of the specific viscosity}


Figure 2 shows the dependence of the specific viscosity $\eta_{\mathrm{sp}}$ on concentration of $\mathrm{Na}-\mathrm{CMC}$ solutions in the PG/water mixture $\left(c_{P G}=80 \%\right)$. In this work the $\eta_{0}$ values were determined by the Cross equation:

$$
\eta=\frac{\eta_{0}}{1+\left(\lambda_{C} \cdot \dot{\gamma}\right)^{m}}
$$

where $\lambda_{c}$ is the characteristic time, and $m$ is the related exponent ${ }^{44}$ (Figures S3-S5, appendix). For $\mathrm{Na}-\mathrm{CMC}_{0.62}$ and $\mathrm{Na}-\mathrm{CMC}_{0.79}$ solutions, zero shear viscosity measurements were carried out in the polymer concentration range from $0.01 \%$ to $1.4 \%$. Above the concentration of $1.4 \%$, there was a violent increase in viscosity, and zero shear viscosity determination was impossible. ${ }^{32}$

For $\mathrm{Na}-\mathrm{CMC}_{1.04}$ solutions two concentration ranges can be distinguished that differ in the slope $\left(\eta_{\mathrm{sp}} \propto c^{a}\right)$. In the first range, the power law exponent is 0.81 , which at high concentrations of $\mathrm{Na}-\mathrm{CMC}_{1.04}$ increases to a value of 2.5 (second range). However, there is no distinct regime with a slope of $\eta_{s p} \propto c^{1.5}$ visible, characteristic for semidilute entangled polyelectrolyte solutions in water. For $\mathrm{Na}-\mathrm{CMC}_{0.62}$ and $\mathrm{Na}-\mathrm{CMC}_{0.79}$ solutions, the slope of experimental points is higher than for $\mathrm{Na}-\mathrm{CMC}_{1.04}$ solutions and is, respectively, 0.89 and 0.83 in the first range, and 3.13 in the second range.

The value of $a=0.81$ for $\mathrm{Na}-\mathrm{CMC}_{1.04}$ is slightly higher than the value of 0.68 given by Lopez et al. $^{45}$ for unentangled semidilute Na-CMC solution in water (theoretical value for polyelectrolyte solutions in good solvent is $0.5) .{ }^{46}$ The beginning of the concentrated regime $\left(c^{* *}\right)$ was identified based on the intersection of curves $\eta_{s p}=f(c)$ in the first and second ranges. The $c^{* *}$ concentrations are $0.42 \% ; 0.51 \%$ and $0.70 \%$ for $\mathrm{Na}-\mathrm{CMC}_{0.62}, \mathrm{Na}-\mathrm{CMC}_{0.79}$ and $\mathrm{Na}-$ $\mathrm{CMC}_{1.04}$, respectively. These values are considerably lower than those obtained for $\mathrm{Na}$ CMC aqueous solutions $(0.82 \% ; 1.15 \%$ and $1.73 \%$ for $\mathrm{Na}-\mathrm{CMC}_{0.62}, \mathrm{Na}-\mathrm{CMC}_{0.79}$ and $\mathrm{Na}$ $\mathrm{CMC}_{1.04}$, respectively). ${ }^{17}$ Additionally, the data published by Różańska and Różański ${ }^{17}$ show that in the case of aqueous solutions of $\mathrm{Na}-\mathrm{CMC}$, the power law exponents above the $c^{* *}$ concentration are higher than for solutions in the PG/water mixture, and up to a polymer concentration of $2.2 \%$ do not depend on DS ( $\eta_{\mathrm{sp}}$ $\propto c^{3.7}$ for DS $0.62 ; 0.78$ and 1.04 ; this value is similar to that given by Lopez et al. ${ }^{45,47}$ for $\mathrm{Na}$ $\left.\mathrm{CMC}_{1.04}\left(\eta_{\mathrm{sp}} \propto c^{3.4 \pm 0.2}\right)\right)$. For aqueous solutions of $\mathrm{Na}-\mathrm{CMC}_{0.7}$ at concentrations greater than $2.2 \%$, the power law exponent increased to a value of about 5.4 (Figure S6, appendix).

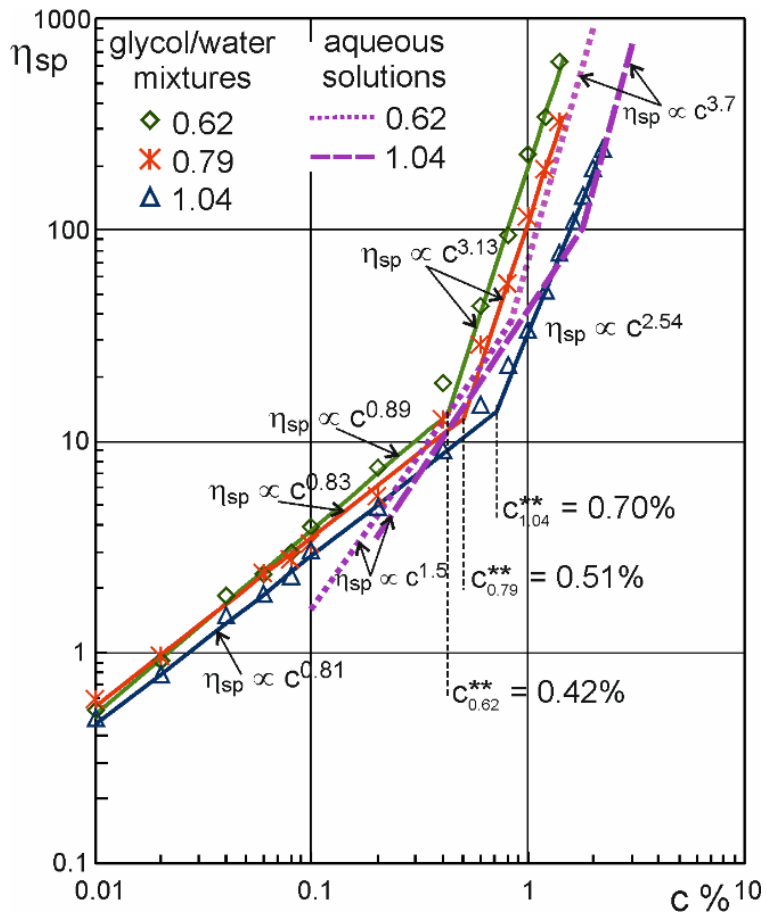

FIGURE 2 Specific viscosity $\eta_{\text {sp }}$ vs. concentration for $\mathrm{Na}-\mathrm{CMC}$ solutions with different degrees of substitution (concentration PG $80 \%$ ). Lines for aqueous solutions of $\mathrm{Na}-\mathrm{CMC}$ (dashed lines) were drawn based on the data published by Różańska and Różański ${ }^{17}$

According to the results of measurements by Lopez et $a .^{47}$, at high DS values, sodium carboxymethylcellulose is molecularly dispersed in water, and the scaling exponent assumes the value of 3.4. Generally, an increase in the power law exponent value above 3.4 suggests the presence of interchain associations in an entangled solution of $\mathrm{Na}-\mathrm{CMC}^{48-51}$. The lower 
values of the power law exponent observed in this study in the concentrated regime may indicate that the PG/water mixture is a "poorer" solvent than pure water. The lower solubility of $\mathrm{Na}-\mathrm{CMC}_{1.04}$ in the poor PG/water solvent induces a change from stretched to collapsed chain conformations, which results in a decrease in the density of entanglements. Since for $\mathrm{Na}-\mathrm{CMC}_{0.62}$ and $\mathrm{Na}-\mathrm{CMC}_{0.79}$ solutions in $\mathrm{PG} /$ water mixtures polymer chain crosslinking occurs, the slope above $c^{* *}$ is larger than for the $\mathrm{Na}-\mathrm{CMC}_{1.04}$ solutions.

\section{Extensional rheology}

\section{Aqueous Na-CMC solutions}

Figure 3 shows apparent extensional viscosity curves for aqueous solutions of $\mathrm{Na}-\mathrm{CMC}$ with different degrees of substitution determined from the filament thinning data following eq (2). The measured surface tension values used to calculate $\eta_{\mathrm{E}, \mathrm{app}}$ were $58.8 \mathrm{mN} / \mathrm{m}, 59.9 \mathrm{mN} / \mathrm{m}$ and $64.2 \mathrm{mN} / \mathrm{m}$ for $\mathrm{Na}-\mathrm{CMC}_{1.04}, \mathrm{Na}-\mathrm{CMC}_{0.9}$ and $\mathrm{Na}-$ $\mathrm{CMC}_{0.62}$, respectively (the surface tension value did not depend on Na-CMC concentration). For the results shown in Figure 3, values of the Bond number were Bo $\ll 0.2$, whereas the Ohnesorge number $\mathrm{Oh} \gg 0.2$, which indicates that the influence of gravity and inertia on capillary thinning could safely be neglected.

The results in Figure 3 show that only for the $\mathrm{Na}$ $\mathrm{CMC}_{1.04}$ solutions at a concentration of $1.4 \%$ the Newtonian plateau at low rates can be distinguished. In all other cases, capillary thinning begins in regime III (and the apparent extensional viscosity decreases with increasing extensional rate). For all $\mathrm{Na}-\mathrm{CMC}_{1.04}$ solutions, at high extensional rates an increase in apparent extensional viscosity at a constant or very narrow range of extension rate $\dot{\varepsilon}$, characteristic of the elastocapillary regime (regime IV), can be observed. Also during capillary thinning of the $\mathrm{Na}-\mathrm{CMC}_{0.79}$ solutions, the apparent extensional viscosity increases at the highest extension rate values.
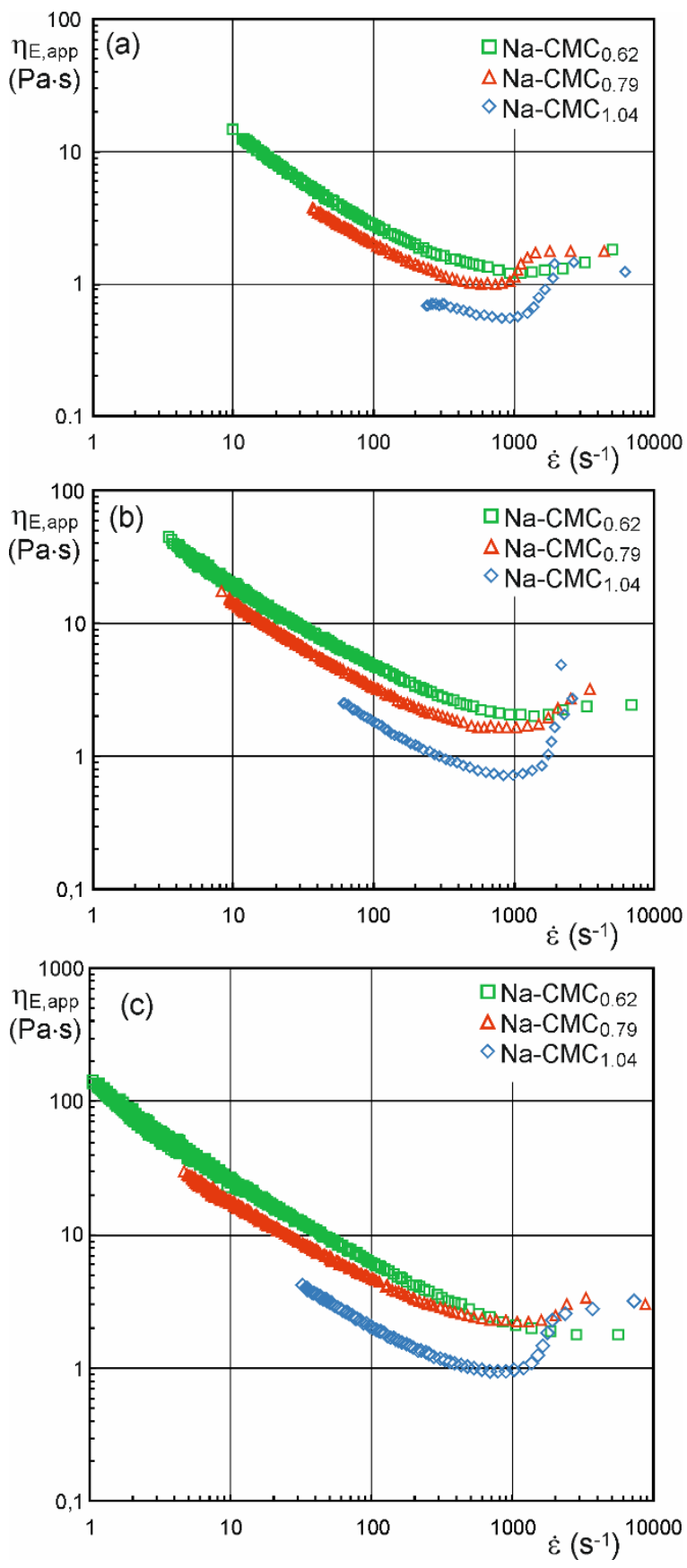

FIGURE 3 Apparent extensional viscosity curves for aqueous solutions of sodium carboxymethylcellulose with concentration $1.4 \%$ (a); $1.8 \%$ (b) and $2.2 \%$ (c)

However, in this case its relative increase is smaller than during capillary thinning of the $\mathrm{Na}$ $\mathrm{CMC}_{1.04}$ solutions, indicating that for $\mathrm{Na}-\mathrm{CMC}$ solutions the elastic contributions to the stresses are comparable to, but not dominating over, the 
viscous contributions. The elastic contributions to the flow at high $\dot{\varepsilon}$ are the lowest for $\mathrm{Na}$ $\mathrm{CMC}_{0.62 \text {. }}$. For these samples, the apparent extensional viscosity at the highest values of $\dot{\varepsilon}$ reaches a constant level, or increases only slightly (the last 3 experimental points).

Figure 4 shows a direct comparison of the time dependency of the evolution of the diameter profiles $D(t) / D_{0}=f(t)$ for Na-CMC solutions with a concentration of $1.8 \%$ and various degrees of substitution.

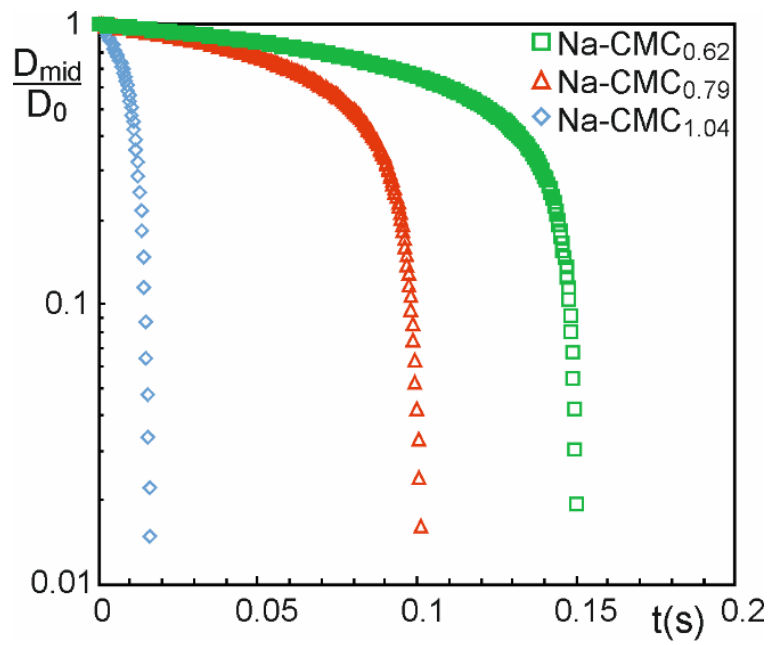

FIGURE $4 D(t) / D_{0}=f(t)$ profile for aqueous solutions of sodium carboxymethylcellulose with a different degrees of substitution

With a decrease of DS, the breakup time $t_{\mathrm{c}}$ is rapidly increasing. The shape of experimental curves, that follows mainly the linear and even faster decay of eqs. (6) and (7), indicates that this is due to the longer time in which visco-capillary thinning occurs (regime III), and the generally higher viscosity of the smaller DS samples in this regime.

The relations between the specific viscosity $\eta_{\mathrm{sp}}$ and the concentration ${ }^{17}$ show that, except for the $1.4 \% \quad \mathrm{Na}-\mathrm{CMC}_{1.04}$ samples, all solution concentrations were higher than $c^{* *}$ (for Na-CMC aqueous solutions $c^{* *}$ is $1.73 \% ; 1.15 \%$ and $0.82 \%$ for degrees of substitution of 1.04, 0.79 and 0.62 , respectively). At $\mathrm{Na}-\mathrm{CMC}$ concentrations higher than $c^{* *}$ there is a relatively strong effect of DS on the zero shear viscosity. At high DS values, sodium carboxymethylcellulose is molecularly dispersed in water ${ }^{45,47}$ and the stronger increase in zero shear viscosity above $c^{* *}$ is associated with the occurrence of entanglements. At low degrees of substitution (DS $<0.82$ ), in aqueous $\mathrm{Na-CMC}$ solutions, the polymer chains are partially aggregated via hydrophobic interactions. ${ }^{45,50}$ This partial aggregation of polymer chains also contributes to an increase of the shear and extensional viscosity, and thus to the extension of time in which visco-capillary thinning dominates, as observed in Figure 4. Moreover, it is probable that the aggregation of polymer chains is the reason for the disappearance of the elastocapillary regime at higher concentrations of $\mathrm{Na}$ $\mathrm{CMC}_{0.62}$. The increase in the number of aggregates with increasing concentration of the polymer results in the fact that capillary thinning is dominated by their disintegration and not the stretching of chains.

It should be noted that the extensional viscosity curves shown in Figure 3 differ qualitatively from those presented in the studies by Jimenez et al. ${ }^{30}$ and Walter et al. $^{31}$ for polyelectrolyte solutions. These authors observed only the occurrence of the strain hardening regime (regime IV) and the strain independent extensional viscosity at late times of thinning. The discrepancies result from a different range of polyelectrolyte concentrations used in their studies. Our measurements were performed for concentrated $\mathrm{Na}-\mathrm{CMC}$ solutions $\left(c>c^{* *}\right)$, while Jimenez et al. ${ }^{30}$ and Walter et al. ${ }^{31}$ used semidilute unentangled and entangled polyelectrolyte solutions.

\section{Na-CMC solutions in PG/water mixture}

The results of CaBER experiments on aqueous solutions of $\mathrm{Na}-\mathrm{CMC}$ discussed in the previous section indicate that DS has an effect on the time to breakup and the capillary thinning in regime IV. Still, the shape of the apparent extensional viscosity curves was qualitatively similar for $\mathrm{Na}-$ CMC with different degrees of substitution. Figures 5 and 6 show the impact of DS on the 
apparent extensional viscosity $\eta_{\mathrm{E}, \mathrm{app}}=f(\dot{\varepsilon})$ for $\mathrm{Na}-\mathrm{CMC}$ solutions in $\mathrm{PG} /$ water mixtures. The surface tension of $\mathrm{Na}-\mathrm{CMC}$ solutions in the PG/water mixture, necessary to calculate $\eta_{\mathrm{E}, \text { app }}$ via eq. (2), depended on the PG concentration only (Table 1).
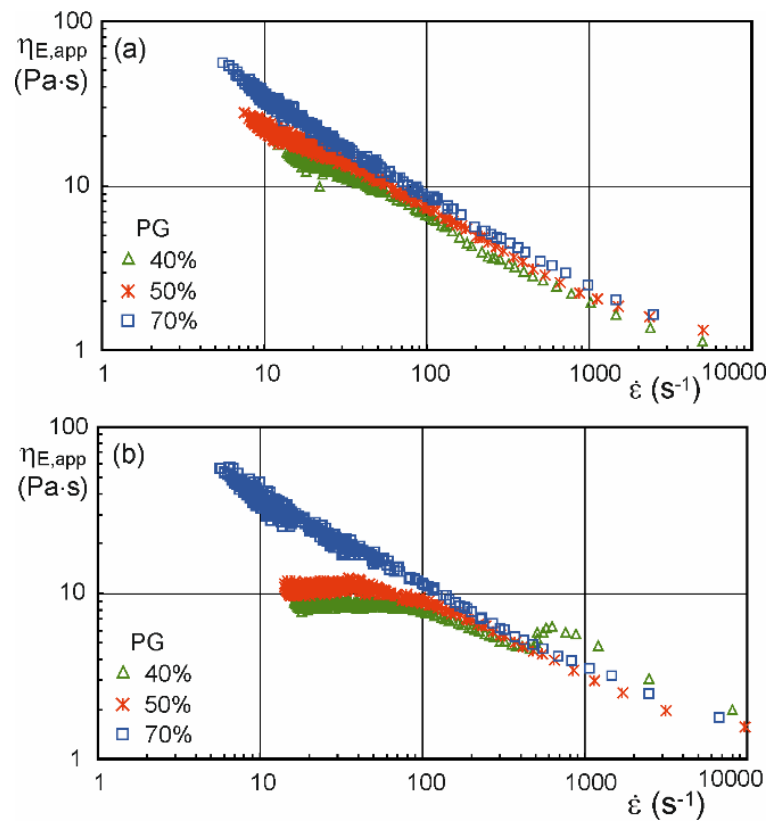

FIGURE 5 Effect of PG concentration on apparent extensional viscosity of $\mathrm{Na}-\mathrm{CMC}_{0.62}$ (a) and $\mathrm{Na}$ $\mathrm{CMC}_{0.79}$ (b) solutions ( $\left.c=1.6 \%\right)$

In this case, the increase in DS to 1.04 leads also to qualitative changes in the shape of the apparent extensional viscosity curves. During the extensional flow of the solutions of $\mathrm{Na}-\mathrm{CMC}_{0.62}$ and $\mathrm{Na}-\mathrm{CMC}_{0.79}$ with a PG concentration $\geq$ of $50 \%$, only visco-capillary thinning occurs. Only for the $\mathrm{Na}-\mathrm{CMC}_{0.79}$ solution with at the lowest PG concentration of $40 \%$, elasto-capillary thinning occurred to a limited extent, and was preceded by regimes II and III.

Similar to the aqueous solutions, also in the $\mathrm{PG} /$ water mixture the high $\mathrm{Na}-\mathrm{CMC}_{1.04}$ shows a markedly different behaviour in comparison to the lower DS samples. In Figure 6 it is possible to distinguish the Newtonian regime at a low extensional rate, immediately followed by an increase in extensional viscosity with increasing $\dot{\varepsilon}$. To identify the reason for this,
Table 1 lists the values of the Bo, Oh, De and Ec numbers, calculated using the initial filament diameter $D_{0}$ for the mid-filament diameter $D_{\text {mid }}$ and the zero shear viscosity $\eta_{0}$. De and Ec were calculated using the terminal relaxation time $\lambda_{0}$ obtained from the LVE data.

$$
\lambda_{0}=\lim _{\omega \rightarrow 0} \frac{G^{\prime}}{G^{\prime \prime} \omega}
$$

Since only for $\mathrm{Na}-\mathrm{CMC}_{1.04}$ solutions at low oscillation frequencies, $G^{\prime} \propto \omega^{2}$ and $G^{\prime \prime} \propto \omega^{1}$ dependencies are met, the terminal relaxation time has been calculated only for solutions of this polymer.

For all $\mathrm{Na}-\mathrm{CMC}$ solutions in the $\mathrm{PG} /$ water mixture the Bond number Bo $<0.2$, whereas the Ohnesorge number $\mathrm{Oh} \gg \mathrm{1}$, thus the differences in the evolution of the extensional viscosity curves is not influenced by inertial and gravitational effects on the capillary thinning. Moreover, the large Deborah number indicates that possible effects of inertia are also smaller than the elastic contribution.

Based on the results of oscillatory measurements performed for $\mathrm{Na}-\mathrm{CMC}_{0.62}$ and $\mathrm{Na}-\mathrm{CMC}_{0.79}$ solutions, there is no possibility to determine the terminal relaxation time, which is why in this case the elasto-capillary number Ec was estimated using the characteristic time of the solutions resulting from the Cross equation.

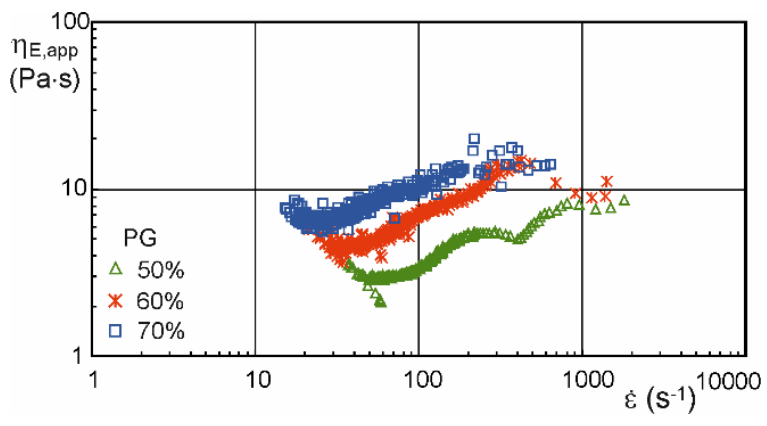

FIGURE 6 Effect of PG concentration on apparent extensional viscosity of $\mathrm{Na}-\mathrm{CMC}_{1.04}$ solutions (c $=1.6 \%)$

The threshold value of $\mathrm{Ec}=4.7$ in the solutions for which the extensional viscosity curves are 
shown in Figure 5 was reached for extension rates ranging from $12 \mathrm{~s}^{-1}$ to $35 \mathrm{~s}^{-1}$, whereas immediately before the filament break-up the Ec values ranged from 61 to 111 . The disappearance of the elasto-capillary thinning regime at such high Ec values can be correlated to the pronounced physical crosslinking of polymer chains.

Table 1. Characteristics of $\mathrm{Na}-\mathrm{CMC}_{1.04}$ in $\mathrm{PG} /$ water mixture solutions

\begin{tabular}{cccccccccc}
\hline $\begin{array}{c}c \\
(\%)\end{array}$ & $\begin{array}{c}C P G \\
(\%)\end{array}$ & $\begin{array}{c}\Gamma \\
(\mathrm{mN} / \mathrm{m})\end{array}$ & $\begin{array}{c}\eta_{0} \\
(\mathrm{~Pa} \cdot \mathrm{s})\end{array}$ & $\begin{array}{c}\lambda_{0} \\
(\mathrm{~s})\end{array}$ & Bo & Ec & Oh & De \\
\hline 1.4 & 80 & 43.0 & 1.59 & 0.101 & 0.041 & 6.60 & 11.6 & 76.7 \\
1.6 & 80 & 43.0 & 2.34 & 0.155 & 0.035 & 7.44 & 17.8 & 132.5 \\
2.0 & 80 & 43.0 & 4.06 & 0.248 & 0.016 & 10.13 & 37.5 & 378.0 \\
2.2 & 80 & 43.0 & 5.02 & 0.319 & 0.025 & 8.48 & 41.6 & 352.3 \\
1.6 & 50 & 48.9 & 0.74 & 0.046 & 0.033 & 7.7 & 5.2 & 40.1 \\
1.6 & 60 & 46.6 & 1.05 & 0.082 & 0.032 & 10.8 & 7.7 & 82.3 \\
1.6 & 70 & 44.4 & 1.50 & 0.12 & 0.022 & 11.2 & 12.4 & 139.3 \\
1.6 & 90 & 41.4 & 3.10 & 0.20 & 0.049 & 6.0 & 22.3 & 134.1 \\
\hline
\end{tabular}

The results of measurements for the $\mathrm{Na}-\mathrm{CMC}_{1.04}$ solutions in the PG/water mixture were surprising. According to the classifications proposed by Clasen et al. ${ }^{6}$, the $\mathrm{Na}-\mathrm{CMC}_{1.04}$ solutions with $\mathrm{Ec}>4.7$, and $\mathrm{Oh}>0.2$ (Table 1) can be considered as a viscoelastic fluid of high viscosity that show the characteristics of dilute and semidilute polymer solutions with a low number of entanglements. ${ }^{6}$ The PG/water mixture is thus a less good solvent for $\mathrm{Na}-\mathrm{CMC}_{1.04}$ than pure water and the expansion of the coils decreases, which results in a reduction in the density of entanglements, and is probably the cause of the disappearance of regime III and the elasto-capillary behaviour in the last stage.

The experimental results obtained for $\mathrm{Na}-\mathrm{CMC}$ solutions in PG/water mixture with different DS are qualitatively consistent with the results obtained by Sharma et al. ${ }^{29}$ for semidilute aqueous solutions of ethyl hydroxyethyl cellulose (EHEC), and its hydrophobically modified analogue (hmEHEC). The semidilute solutions of hmEHEC exhibit extensional thinning, while the unmodified bare chains of
EHEC display an increase in extensional viscosity, up to a plateau value. The hydrophobic groups on the polymer chains promote transient interchain associations, which leads to aggregate formation and enhanced viscosities. During thinning, the aggregates break down, which results in a decrease in apparent extensional viscosity with increasing extension rate.

Figure 7 shows the dependencies of the midfilament diameter evolution as a function of time for $\mathrm{Na}-\mathrm{CMC}_{1.04}$ solutions with a variable polymer concentration and a constant concentration of $80 \%$ PG (Figure 7a), and with a variable concentration of PG and a constant polymer concentration of $1.6 \%$ (Figure $7 \mathrm{~b}$ ). The shape of curves does not allow determining unambiguously the time range in which the exponential filament decay occurs, hence equation (11) cannot be used to determine the extensional relaxation time. However, the dependence $D_{\text {mid }} / D_{0}=f(t)$ is well fitted by the lines described by equation (13) (solid lines plotted in Figure 7). Hence, based on the determined value of the constant $B$, the 
extensional relaxation time values $\lambda_{\mathrm{E}}$ were calculated.
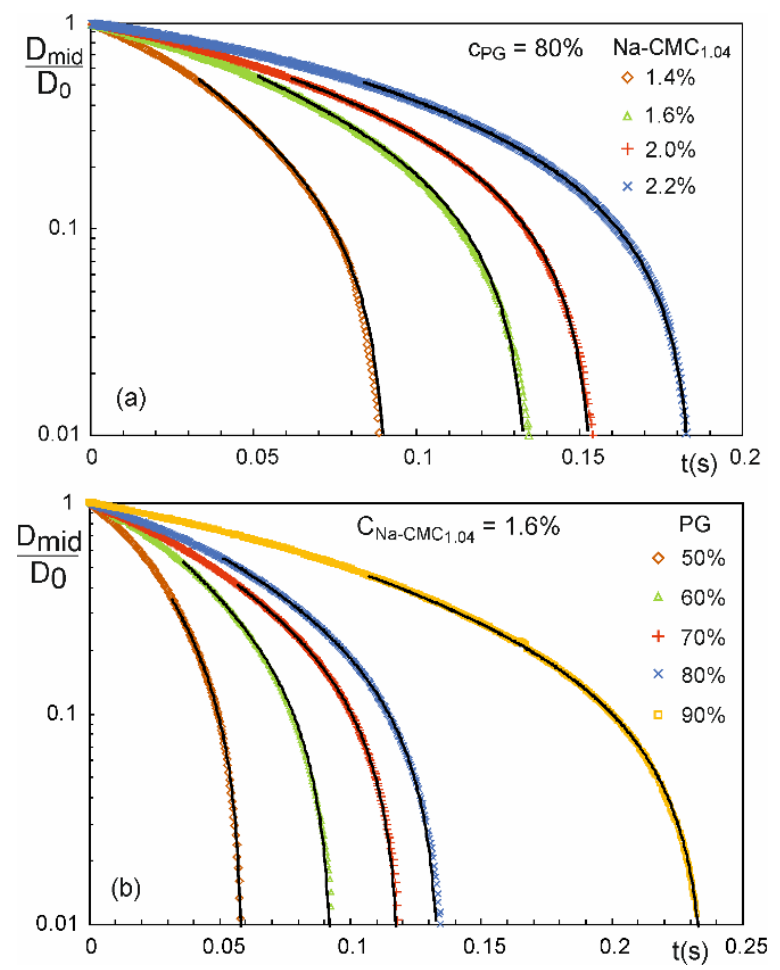

FIGURE 7 Effect of Na-CMC (a) and PG (b) concentration on mid-filament diameter evolution with time.

Figure 8 shows the dependencies of $\lambda_{E}$ and $\lambda_{0}$ on the $\mathrm{Na}-\mathrm{CMC}_{1.04}$ and $\mathrm{PG}$ concentrations. The extensional relaxation time is much shorter than the terminal relaxation time, which is characteristic for semidilute and concentrated polymer solutions $\left(\right.$ Clasen $\left.^{35}\right)$. The increase in PG concentration causes the growth of $\lambda_{\mathrm{E}}$ and $\lambda_{0}$, and the scaling of the curves with concentration $\lambda_{\mathrm{E}}=f\left(c_{\mathrm{PG}}\right)$ and $\lambda_{0}=f\left(c_{\mathrm{PG}}\right)$ are similar $\left(\lambda_{\mathrm{E}} \propto c_{P G}^{2.55} \lambda_{0}\right.$ $\left.\propto c_{P G}^{2.46}\right)$, indicating that the change in solvent viscosity causes a similar increase in the relaxation time for both, extensional and shear flows. with the Cross equation. However, the data in Figure 7a shows that the scaling of $\lambda_{E}$ and $\lambda_{0}$ with $\mathrm{Na}-\mathrm{CMC}_{1.04}$ concentration is different. The power law exponent is $2.47 \pm 0.11$ for $\lambda_{0}$ and $1.56 \pm 0.17$ for $\lambda_{E}$. These results are qualitatively consistent with data published in literature for the entangled solution of other polymers. In the range of the unentangled semidilute and the entangled semidilute the extensional relaxation time $\lambda_{E}$ increases with an increasing polymer concentration, while at the same time $\lambda_{\mathrm{E}}<\lambda_{0}{ }^{25,28,52,53}$ and the related slope of $\lambda_{\mathrm{E}}=f(\mathrm{c})$ is smaller than for $\lambda_{0}=f(\mathrm{c})$ (Arnolds et al. ${ }^{52}$ ). Figure 8 also shows values of the characteristic time $\lambda_{c}$ of the solutions, obtained by fitting the flow curves with the Cross equation.
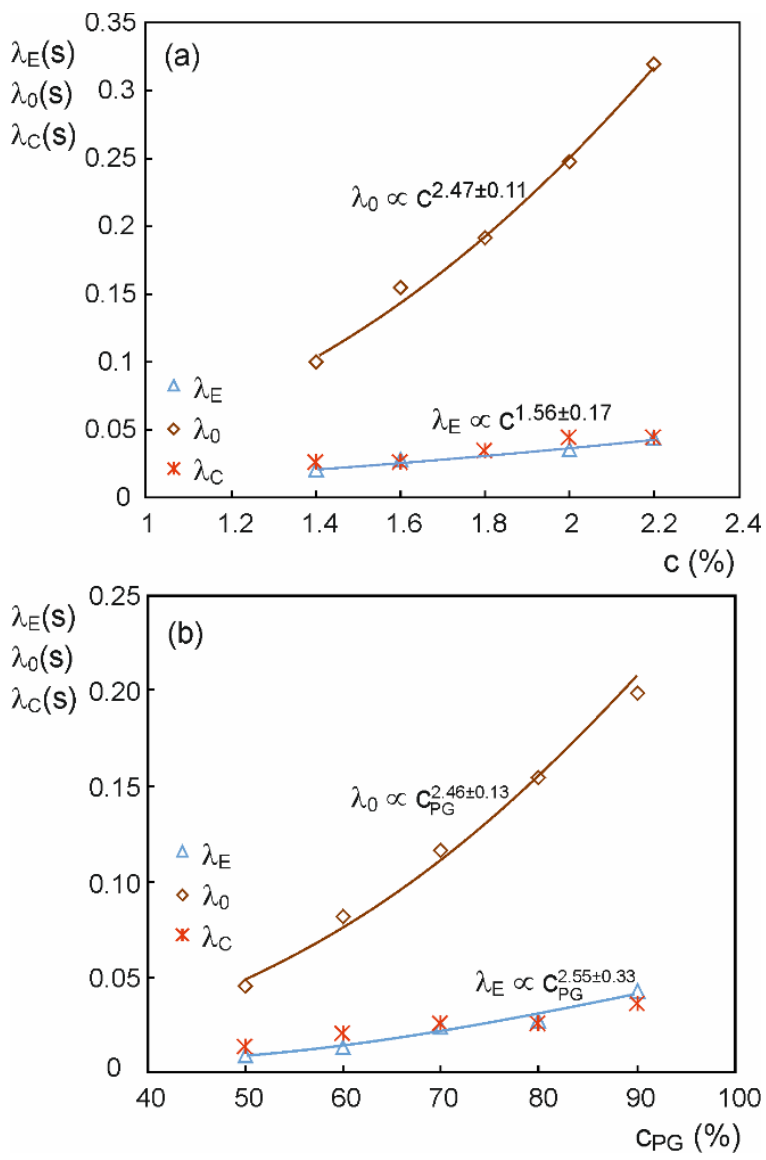

FIGURE 8 Relaxation times $\lambda_{0}, \lambda_{\mathrm{E}}$ and characteristic time $\lambda_{c}$ as a function of $\mathrm{Na}-\mathrm{CMC}_{1.04}$ (a) and PG (b) concentration

The values of characteristic time $\lambda_{c}$ are close to the estimated values of the extensional relaxation time $\lambda_{E}$. Similar results were reported for a set of concentrated alginate solutions ${ }^{54}$ and mucilage droplets. ${ }^{55}$ On the other hand, much higher values of $\lambda_{C}$ than $\lambda_{E}$ for entangled ultrahigh-molecular weight alginates in $\mathrm{NaCl}$ solutions and aqueous guar gum derivative solutions were observed. ${ }^{25,56}$ Those higher 
values were attributed to the presence of aggregates in the solution (superstructures/hyperentanglements) that result in an increase in the zero shear viscosity, a decrease in $\dot{\gamma}_{c}$ and thus an increase in $\lambda_{c}$. In $\mathrm{PG} /$ water solutions, the $\mathrm{Na}-\mathrm{CMC}_{1.04}$ chains do not crosslink, and the intersection of the $\mathrm{G}^{\prime}$ and G' modulus occurs at high oscillation frequency rates, which indicates a low density of entanglements. The onset of the shear thinning regime is thus mainly related to the alignment of polymer chains in the flow direction. Similar values of $\lambda_{C}$ and $\lambda_{E}$ for $\mathrm{Na}-\mathrm{CMC}_{1.04}$ solutions may be related to the similarity of stress relaxation during stretching in extensional flow and alignment in shear flow of polymer chains.

\section{CONCLUSIONS}

This paper presents results of CaBER experiments for semidilute solutions of $\mathrm{Na}-\mathrm{CMC}$ with degrees of substitution in the range from 0.62 to 1.04 in distilled water and PG/water mixtures. The partial aggregation of $\mathrm{Na}-\mathrm{CMC}$ chains with a low degree of substitution, which occurs in aqueous solutions, induces an increase in shear and apparent extensional viscosity, as well as a marked rise of the break-up time, and at higher concentrations of $\mathrm{Na}-\mathrm{CMC}_{0.62}$ it leads to the disappearance of the elasto-capillary regime.

The rheological properties of $\mathrm{Na}-\mathrm{CMC}$ solutions in $\mathrm{PG} /$ water mixture are determined by the solubility of the polymer and the crosslinking of chains. The disappearance of the elasto-capillary regime during the filament thinning of $\mathrm{Na}-\mathrm{CMC}$ solutions in PG/water mixture with low DS $(0.62$ and 0.79) is attributed to the physical crosslinking of polymer chains. In the absence of this crosslinking, the lower solubility of $\mathrm{Na}$ $\mathrm{CMC}_{1.04}$ in the poor PG/water solvent induces a reduction of the coil size, which results in a decrease in the density of entanglements. For these reasons, the capillary thinning of filaments of the $\mathrm{Na}-\mathrm{CMC}_{1.04} / \mathrm{PG} /$ water system progresses in a similar manner as for semidilute polymer solutions with a low number of entanglements. In this case, a Newtonian thinning regime at a low extensional rate is noted, and followed directly by an increase in extensional viscosity with increasing $\dot{\varepsilon}$.

The extensional relaxation time of $\mathrm{Na}-\mathrm{CMC}_{1.04}$ solutions in PG/water mixture was markedly shorter than the terminal relaxation time $\lambda_{0}$, which is characteristic for unentangled semidilute and entangled semidilute polymer solution. On the other hand, the values of the characteristic time of the solution $\lambda_{c}$ resulting from the Cross model were comparable to the values of the extensional relaxation time. Similar values of $\lambda_{C}$ and $\lambda_{E}$ may be related to the similarity of stress relaxation during stretching in extensional flow and alignment in shear flow of $\mathrm{Na}-\mathrm{CMC}_{1.04}$ chains.

\section{ACKNOWLEDGEMENTS}

This work was supported by the Polish Ministry of Science and Higher Education (Grant no. 03/32/SBAD/0902).

$\mathrm{KV}$ and $\mathrm{CC}$ acknowledge financial support by the Bijzonder Onderzoeksfonds KU Leuven (GOA 15/007).

\section{REFERENCES}

1. R. Barbucci, A. Magnani, M. Consumi, Macromolecues, 3, 2000, 7475.

2. H. Kono, K. Oshima, H. Hashimoto, Y. Shimizu, K. Tajima, Carbohydr. Polym., 146, 2016, 1.

3. W. Li, B. Sun, P. Wu, Carbohydr. Polym., 78, 2009, 454.

4. S. Różańska, L. Broniarz-Press, J. Różański, P T. Mitkowski, M. Ochowiak, S. Woziwodzki, Food Hydrocoll., 32, 2013, 130.

5. S. Zell, S. Gier, S. Rafai, C. Wagner, J. NonNewton. Fluid Mech., 165, 2010, 1265.

6. C. Clasen, P. M. Phillips, L. Palangetic, J. Vermant, AICHE J., 58, 10, 2012, 3242.

7. M. P. Escudier, F. Presti, S. Smith, J. NonNewton. Fluid Mech., 81(3), 1999, 197.

8. A. Kloziński, P. Jakubowska, Polym. Eng. Sci., 2018, 1. 
9. S. Nigen, K. Walters, J. Non-Newton. Fluid Mech., 102, 2002, 343.

10. M. Zatloukal, J. Vlček, C. Tzoganakis, P. Sáha, J. Non-Newton. Fluid Mech., 107, 2002, 13.

11. M. Ochowiak, L. Broniarz-Press, S. Rozanska, J. Rozanski, J. Ind. Eng. Chem., 18(6), 2012, 2028.

12. P. T. Mitkowski, W. http://www.sciencedirect.com/science/arti cle/pii/S0263876216300193 cor0005mailto:piotr.mitkowski@put.pozna n.plSzaferski, mailto:waldemar.szaferski@put.poznan.pl Chem. Eng. Res. Des., 109, 2016, 618.

13. S. Różańska, Practical Aspects of Chemical Engineering. Springer, 2018, pp. 377-393.

14. J. Różański, S. Różańska, P. Mitkowski, W. Szaferski, P. Wagner, A. Marecka-Migacz, A. Kuczora, Chem. Ind., 97(12), 2018,1000 (in Polish).

15. T. Sochi, J. Polym. Sci., Part B: Polym. Phys., 48, 2010, 2437.

16. S. Różańska, Chem. Eng. Equip., 52(6), 2013, 557 (in Polish).

17. S. Różańska, J. Różański, Soft Mater., 15, 4, 2017, 302.

18. H. Choi, J. R. Mitchell, S. R. Gaddipati, S. E. Hill, B. Wolf, Food Hydrocoll., 40, 2014, 71.

19. G. H. Zhao, N. Kapur, J. T. Guthrie, Eds.; P. A. Williams, G. O. Phillips, Royal Society of Chemistry, UK. 2012; pp 391-398.

20. S. J. Haward, V. Sharma, C. P. Butts, G. H. McKinley, S. S. Rahatekar, Biomacromolecules, 13(5), 2012, 1688.

21. A. Jaishankar, M. Wee, L. Matia-Merino, K. T. Kelvin, G. H. McKinley, Carbohydr. Polym., 123, 2015, 136.

22. M. R. Duxenneuner, P. Fischer, E. J. Windhab, J. J. Cooper-White, Biomacromolecules 9, 2008, 2989.

23. A. Ö. Bingöl, D. Lohmann, K. Püschel, W. M. Kulicke, Biorheology 47, 2010, 205.

24. A. Bourbon, A. Pinheiro, C. Ribeiro, C. Miranda, J. Maia, J. Teixeira, A. A. Vicente, Food Hydrocoll., 24(2-3), 2010, 184.
25. D. Szopinski, U. A. Handgeb, W. M. Kulicke, V. Abetz, G. A. Luinstra, Carbohydr. Polym., 136, 2016, 834.

26. M. D. Torres, B. Hallmark, D. I. Wilson, Food Hydrocoll., 40, 2014, 85.

27. S. S. Vadodaria, R. J. English, Cellulose, 23, 2016, 339.

28. R. De Dier, W. Mathues, C. Clasen, Macromol. Mater. Eng., 298, 2013, 944.

29. V. Sharma, S. J. Haward, J. Serdy, B. Keshavarz, A. Soderlund, P. ThrelfallHolmes, G. H. McKinley, Soft Matter 11, 2015, 3251.

30. L. N. Jimenez, J. Dinic, N. Parsi, V. Sharma, Macromolecules 51, 2018, 5191.

31. A. V. Walter, L. N. Jimenez, J. Dinic, V. Sharma, K. A. Erk, Rheol. Acta 58, 2019, 145.

32. P. Komorowska, S. Różańska, J. Różański, Cellulose 24, 10, 2017, 4151.

33. S. L. Anna, G. H. McKinley, J. Rheol., 45, 2001, 115.

34. D. C. Vadillo, W. Mathues, Ch. Clasen, Rheol. Acta, 51, 2012, 755.

35. C. Clasen, Korea-Aust. Rheol. J., 22(4), 2010, 331.

36. D. T. Papageorgiou, Physics of Fluids, 7(7), 1995, 1529.

37. G. H. McKinley, A. Tripathi, J. Rheol., 44, 2000, 653.

38. P. Doshi, R. Suryo, O. E. Yildirim, G. H. McKinley, O. A. Basaran, J. Non-Newton. Fluid Mech., 113(1), 2003, 1.

39. V. M. Entov, E. J. Hinch, J. Non-Newton. Fluid Mech., 72, 1997, 31.

40. M. Chellamuthu, J. P. Rothstein, J. Rheol., 52(3), 2008, 865.

41. W. Miller, C. Clasen, J. P. Rothstein, Rheol. Acta, 48, 2009, 625.

42. ASTM, 1961.

43. H. Toğrul, N. Arslan, Carbohydr. Polym., 54(1), 2003, 73.

44. Y. Feng, G. Han, L. Zhang, S. B. Chen, T. S. Chung, M. Weber, C. Staudt, C. Maletzko, Polymer 99, 2016, 72.

45. C. G. Lopez, R. H. Colby, P. Graham, J. T. Cabral, Macromolecules 50, 2017, 332.

46. A. V. Dobrynin, M. Rubinstein, Prog. Polym. Sci., 30, 2005, 1049. 
47. C. G. Lopez, S. E. Rogers, R. H. Colby, P. Graham, J. T. Cabral, J. Polym. Sci., Part B: Polym. Phys., 53, 2015, 492.

48. J.S. Behra, J. Mattsson, O. J. Cayre, E. S. J. Robles, H. Tang, T. N. Hunter, ACS Appl. Polym. Mater., 1, 2019, 344.

49. C. Barba, D. Montané, X. Farriol, J. Desbrières, M. Rinaudo, Cellulose 9, 2002, 327.

50. C. G. Lopez, R. H. Colby, J. T. Cabral, Macromolecules 51, 8, 2018, 3165.

51. C. G. Lopez, W. Richtering, Cellulose 26, 2019, 1517.

52. O. Arnolds, H. Buggisch, D. Sachsenheimer, N. Willenbacher, Rheol. Acta, 49, 2010, 1207.
53. S. Kheirandish, I. Guybaidullin, W. Wohlleben, N. Willenbacher, Rheol. Acta, 47, 2008, 999.

54. C. Rodríguez-Rivero, L. Hilliou, E. M. Martín del Valle, M .A. Galán, Rheol. Acta, 53, 2014, 559.

55. P. Erni, M. Varagnat, C. Clasen, J. Cresta, W. M. McKinley, Soft Matter 7, 2011, 10889.

56. H. Storz, U. Zimmermann, H. Zimmermann, W. M. Kulicke, Rheol. Acta, 49, 2010, 155. 


\section{GRAPHICAL ABSTRACT}

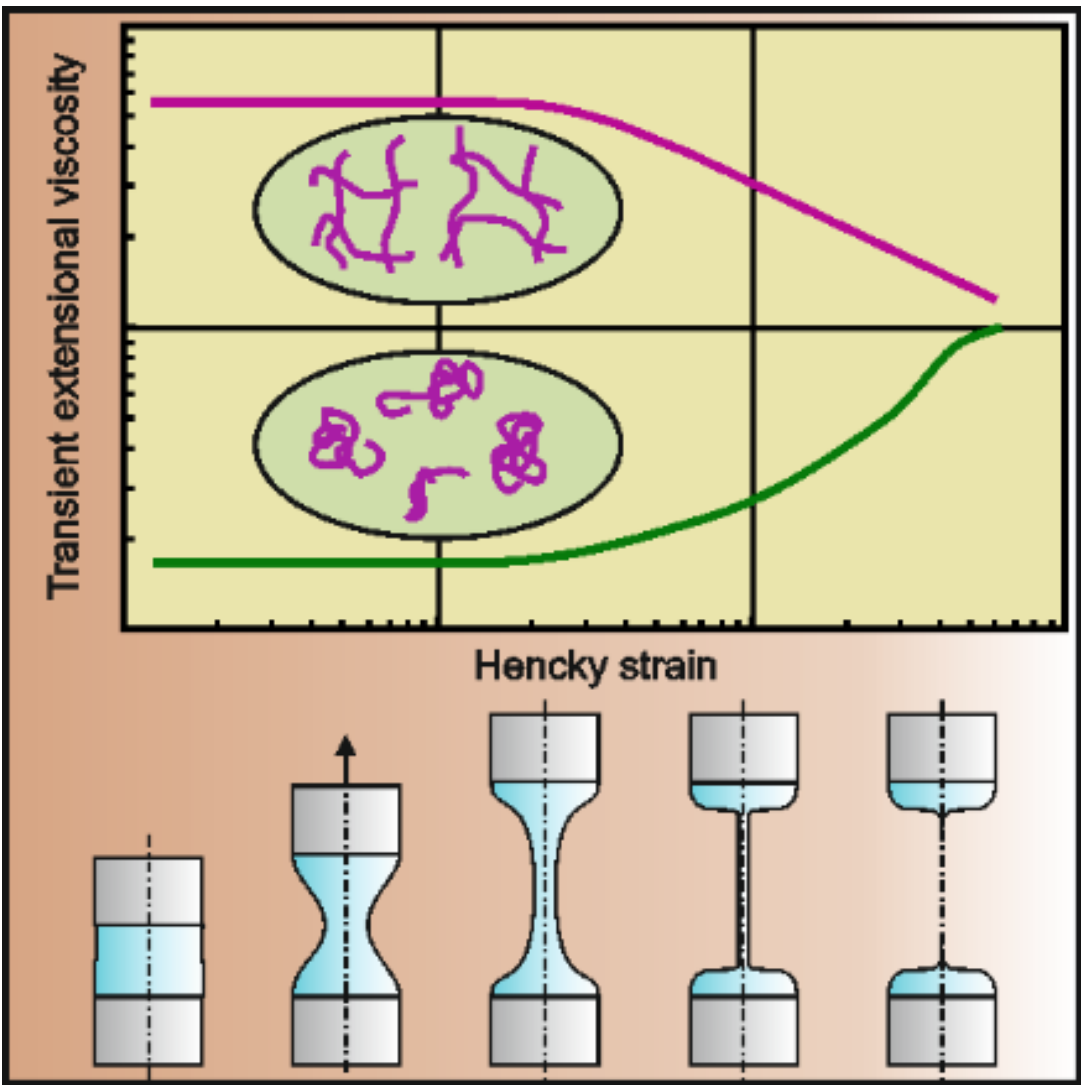

\section{AUTHOR NAMES}

Sylwia Różańska, Karel Verbeke, Jacek Różański, Christian Clasen, Patrycja Wagner

\section{TITLE}

\section{Capillary breakup extensional rheometry (CaBER) of sodium carboxymethylcellulose solutions in water and propylene glycol/water mixtures}

TEXT

The CaBER rheometer is used to measure extensional viscosity of low-viscosity fluids. With the use of that type of rheometer, the apparent extensional viscosity and the extensional rate can be determined using the time-dependent changes in the fiber midpoint diameter and with performing the independent measurement of surface tension. This paper presents measurement results obtained with use of CaBER rheometer for semi diluted $\mathrm{Na}-\mathrm{CMC}$ solutions in water and propylene glycol/water mixtures. 